\title{
ERRATUM
}

\section{MicroRNA-146a directs the symmetric division of Snail-dominant colorectal cancer stem cells}

Wei-Lun Hwang, Jeng-Kae Jiang, Shung-Haur Yang, Tse-Shun Huang, Hsin-Yi Lan, Hao-Wei Teng, Chih-Yung Yang, Ya-Ping Tsai, Chi-Hung Lin, Hsei-Wei Wang and Muh-Hwa Yang

Nat. Cell Biol. 16, 268-280 (2014); published online 23 February 2014; corrected after print 26 February 2014

In the version of this Article originally published, the labels of the key in the upper panel of Fig. 7h should have read 'HT29-Vec' (black) and 'HT29-146a' (grey). This error has now been corrected in the online versions of the Article.

\section{CORRIGENDUM}

\section{Rho-directed forces in collective migration}

\section{Peter Friedl, Katarina Wolf and Mirjam M. Zegers}

Nat. Cell Biol. 16, 208-210 (2014); published online 28 February 2014; corrected after print 14 March 2014

In the version of this News and Views article originally published, the author affiliations were incorrect. The correct affiliations are as follows: Peter Friedl, Katarina Wolf and Mirjam M. Zegers are in the Department of Cell Biology, Radboud University Nijmegen Medical Centre, 6500 HB Nijmegen, The Netherlands. Peter Friedl is at the David H. Koch Center for Applied Research of Genitourinary Cancers, Department of Genitourinary Medical Oncology, The University of Texas MD Anderson Cancer Center, Houston, Texas, 77030, USA. This has been corrected in all online versions of this News and Views article. 